\title{
The Effect of Hypnotics on Sleep Quality and Cognitive Function in Patients with Brain Tumors
}

\author{
Min Cheol Chang, ${ }^{1}$ Min Ho Chun ${ }^{2}$ \\ Department of Rehabilitation Medicine, 'College of Medicine, Yeungnam University, Daegu, Korea \\ Department of Rehabilitation Medicine, ${ }^{2}$ Asan Medical Center, University of Ulsan College of Medicine, Seoul, Korea
}

Objective : We investigated the effect of hypnotics on sleep quality, cognitive function, and depressive mood in patients with insomnia following brain tumor resection.

Methods : From patients who underwent brain tumor resection, we recruited 10 patients with insomnia who received hypnotics for more than 1 week during a 3-week follow-up period (insomnia group). We also recruited 12 control patients with brain tumors but without insomnia (control group). We evaluated sleep quality at baseline and 3 weeks later using the Insomnia Severity Index (ISI), the Pittsburgh Sleep Quality Index (PSQI), the Stanford Sleepiness Scale (SSS), and the Epworth Sleepiness Scale (ESS) and investigated cognitive function and depression using the Computerized Neuropsychological Test and the Beck Depression Inventory (BDI).

Results : At baseline, SSS, ISI, PSQI, and BDI scores were significantly higher and visual continuous performance test (VCPT) and auditory continuous performance test (ACPT) scores were significantly lower in the insomnia than in the control group. Three weeks later, the patients who had received hypnotics had significantly higher ISI, PSQI, ESS, VCPT, ACPT, visual span forward and backward, and visual recognition test scores, and significantly lower BDI scores.

Conclusion : Quality of sleep in patients with insomnia following brain tumor resection was initially poor but improved significantly after taking hypnotic medication. Further, the hypnotic medications appeared to contribute to the amelioration of cognitive impairments and depressive moods in patients who previously underwent brain tumor resection. We thus recommend the use of hypnotics for patients with brain tumors with insomnia.

Key Words : Hypnotics and sedatives · Sleep initiation and maintenance disorders · Cognition · Depression · Brain neoplasms.

\section{INTRODUCTION}

Although insomnia is one of the most common complaints in patients with brain lesions, little is known about insomnia in these patients ${ }^{26}$. Lesions in the brain areas associated with sleep, such as the hypothalamus, brainstem, and basal forebrain, which are caused by the local effects of a brain tumor or by treatment procedures including surgery and irradiation, can lead to sleep disturbances ${ }^{31}$. Furthermore, sleep disturbances can persist in brain tumor survivors for many years af-

\footnotetext{
- Received : February 21, 2019 •Revised : May 2, 2019 •Accepted : May 19, 2019

- Address for reprints : Min Ho Chun

Department of Rehabilitation Medicine, Asan Medical Center, University of Ulsan College of Medicine, 88 Olympic-ro 43-gil, Songpa-gu, Seoul 05501, Korea Tel : +82-2-3010-3800, Fax : +82-2-3010-6964, E-mail : mhchun0@gmail.com, ORCID : https://orcid.org/0000-0001-8666-7225
} 
ter diagnosis and completion of treatment, making it one of the most persistent problems experienced by patients with brain tumors ${ }^{12}$. Previous studies have documented that although sleep disturbances are common in these patients, the problem has been neglected ${ }^{12)}$. Sleep disturbances are associated not only with reduced cognitive function but also with psychological and physical problems, which can interfere with recovery ${ }^{33)}$. Therefore, early treatment of insomnia is important to maintain active rehabilitation management of patients with brain tumors.

Several forms of treatment for insomnia have been developed, and hypnotic medications have been most frequently prescribed in inpatient rehabilitation units because of their rapid effect on sleep disturbances ${ }^{17,30)}$. Typically, hypnotics are divided into benzodiazepine and non-benzodiazepine drugs. All hypnotics induce and maintain sleep, thus improving the sleep quality of patients with insomnia. However, as an adverse effect, a decline in cognition has been reported in several studies in which members of the general population were administered hypnotics ${ }^{16,28)}$. Hypnotics may thus affect the cognitive function of patients with brain tumors, but the effect has not clearly been demonstrated to date.

In this study, we investigated changes in sleep patterns following administration of hypnotics. In addition, we attempted to determine whether the administration of hypnotics disturbs the cognitive recovery of patients with insomnia following brain tumor resection.

\section{MATERIALS AND METHODS}

\section{Patients}

The study protocol described here was approved by the Ethics Committee of Asan Medical Center, and written informed consent was obtained from each participant. We enrolled patients with a primary diagnosis of brain tumor who were transferred as inpatients to the Department of Rehabilitation Medicine at Asan Medical Center, following their brain tumor resection, between August 2011 and July 2012. A brain tumor was defined as a primary or metastatic lesion, as revealed by computed tomography or magnetic resonance imaging of the brain and confirmed by a pathological diagnosis of the biopsy following surgical resection. All benign and malignant tumors were included. To be enrolled, patients were required to be able to follow simple commands, as determined by a score of 24 or higher on the Mini-Mental State Examination (MMSE). Patients were excluded if they were medically unstable, unable to read, or unable to answer questions because of severe aphasia, apraxia, blindness, deafness, cognitive impairment, or neglect. Patients taking hypnotics (prior to our study), neuroleptics, antipsychotics, anti-epileptics, $\mathrm{H}_{2}$-blockers, or any other potentially sedating drug were also excluded.

No universal tool exists for the diagnosis of insomnia after a brain tumor. Insomnia was therefore diagnosed in patients who underwent brain tumor surgery if at least one of following criteria defined in previous reports of insomnia in the general populations was met ${ }^{7,23)}$. First, a total sleep time less than or equal to 360 minutes; second, a sleep latency greater than 30 minutes; or third, four or more awakenings from sleep during any one night. Patients diagnosed with insomnia were administered hypnotics, prescribed by individual physicians. In addition, individualized rehabilitation programs were provided. Every patient received 1-hour sessions of conventional physical and occupational therapy six times per week for 3 weeks. After 3 weeks, patients who required hypnotics for more than 1 week were classified into the Insomnia group. As controls, we recruited patients with brain tumors who did not have insomnia and were not taking any hypnotic medication.

\section{Measurements}

The Insomnia Severity Index (ISI) ${ }^{2)}$ and the Pittsburgh Sleep Quality Index (PSQI) ${ }^{5}$ were used to evaluate sleep disturbance and quality. The ISI is a brief self-reporting survey instrument measuring the patient's perception of insomnia. It targets the subjective symptoms and the consequences of insomnia, as well as the degree of concern or distress caused by those difficulties. The ISI comprises seven items assessing the severity of sleep onset and sleep maintenance difficulties (both nocturnal and early morning awakenings), satisfaction with the current sleep pattern, interference with daily functioning, noticeability of impairment attributed to sleep problems, and the degree of distress or concern caused by the sleep problems. Each item is rated on a $0-4$ scale, with the total score ranging from 0 to 28 . A higher score suggests more severe insomnia. The PSQI is a 19-item self-rated questionnaire for evaluating subjective sleep quality over the previous month. The 19 questions are combined into seven clinically- 
derived component scores, each weighted equally, and scored from 0 to 3. The seven components are composed of subjective sleep quality, sleep latency, sleep duration, habitual sleep efficiency, sleep disturbances, use of sleeping medication, and daytime dysfunction. These component scores are added to obtain a global score ranging from 0 to 21, with higher scores indicating worse sleep quality.

Daytime sleepiness was evaluated using the Stanford Sleepiness Scale (SSS) ${ }^{15)}$ and the Epworth Sleepiness Scale (ESS) ${ }^{8)}$. The SSS consists of a single seven-point scale. It ranges from 1 (feeling active and vital; alert; wide awake) to 7 (almost in reverie; sleep onset soon; lost struggle to remain awake). The ESS consists of eight self-rated items, each scored from $0-3$, that measure a subject's habitual "likelihood of dozing or falling sleep" in common situations of daily living. Values of SSS $\geq 3$ and values of ESS $>9$ are considered to be indicative of significant daytime sleepiness.

The MMSE and the Computerized Neuropsychological Test (CNT; MaxMedica, Seoul, Korea) were used to assess cognitive function. The CNT was initially developed to evaluate cognitive function of Korean adults ${ }^{14}$. The reliability and validity of the CNT have been demonstrated previously ${ }^{14)}$. The CNT contains the following six subtests : 1) a visual continuous performance test (VCPT); 2) an auditory continuous performance test (ACPT); 3) digit span forward and backward tests (DSFT and DSBT); 4) visual span forward and backward tests (VSFT and VSBT), for assessing attention; 5) an auditory verbal learning test (AVLT); and 6) a visual recognition test (VRT) for measuring memory function. All of the evaluations were performed both at baseline and after 3 weeks. Examiners were blinded to the identity and symptoms of each patient. The VCPT and ACPT were based on Conner's continuous performance test ${ }^{10)}$. The DSFT and DSBT were derived from the Wechsler Adult Intelligence Scale-Neuropsychological Inventory ${ }^{6,20)}$, and the VSFT and VSBT were based on Corsi's block-tapping test ${ }^{4,21)}$. The AVLT was derived from Rey's AVLT $^{27}$. The VRT was similar to the AVLT, but figures were used instead of words.

For evaluating depressive symptoms, the Beck Depression Inventory (BDI), a self-report questionnaire containing 21 items, was used. BDI scores range from 0 to 63 , with higher BDI scores indicating more depression ${ }^{13)}$.

\section{Statistical analysis}

SPSS version 22.0 statistical software (SPSS Inc., Chicago, IL, USA) was used for statistical analysis. The Mann-Whitney U test was used to compare the Insomnia and Control groups in terms of general patient characteristics and regarding the results of the following tests: ISI, PSQI, SSS, ESS, MMSE, CNT, and BDI. The Wilcoxon signed rank test was used to compare baseline and follow-up data for the Control and Insomnia groups. Any association with a $p$ value less than 0.05 was considered to be statistically significant.

\section{RESULTS}

\section{Demographic and disease-related characteristics of the patients}

Of 25 patients who were confirmed to have a brain tumor, 22 patients were enrolled in this study, and 10 were diagnosed with insomnia. Three patients were excluded because they took hypnotics for less than 1 week. Therefore, 10 patients were assigned to the insomnia group, and 12 patients were assigned to the control group. None of the included patients had a history of seizures. The demographic characteristics of the patients in both groups are shown in Table 1. There were no significant differences in sex, age, time since operation, history of radiation therapy, or Motricity Index ${ }^{19)}$ between the Control and Insomnia groups (Mann-Whitney U test, $p>0.05$ ). The following hypnotics were administered for more than 1 week to patients in the insomnia group during the 3 -week follow-up course of the study : seven patients received $10 \mathrm{mg}$ of zolpidem and three patients received $25 \mathrm{mg}$ of trazodone. Patients in the control group were administered no hypnotic medications.

\section{Sleepiness and quality of sleep}

Baseline mean scores for the ISI, PSQI, and SSS were significantly higher in the Insomnia group than in the control group (Mann-Whitney U test, $p<0.05$ ) (Table 2). The ISI, PSQI, and ESS scores in the insomnia group were significantly improved after hypnotic medication, compared with baseline scores (Wilcoxon signed rank test, $p<0.05$ ) (Table 2).

\section{Cognitive function}

There was no significant difference in MMSE scores be- 
tween the insomnia and control groups, either at baseline or at the 3-week follow-up (Mann-Whitney U test, $p>0.05$ ). Significant improvement in MMSE scores was observed between baseline and the follow-up in both groups (Wilcoxon signed rank test, $p<0.05$ ) (Table 3).

During the baseline CNT assessment, the mean scores for VCPT and ACPT in the Insomnia group were significantly lower than the Control group scores (Mann-Whitney U test, $p<0.001)$. However, there were no differences in the mean scores of the other tests between the two groups at baseline (Mann-Whitney U test, $p>0.05$ ). After 3 weeks, in the Insomnia group, the mean scores on the VCPT, ACPT, and VSFT were significantly higher, with more figures being recalled during the first and fifth VRT trials (Wilcoxon signed rank

Table 1. Demographic characteristics of all subjects

\begin{tabular}{|c|c|c|c|}
\hline & Insomnia group & Control group & $p$-value \\
\hline $\operatorname{Sex}(M: F)$ & $4: 6$ & $3: 9$ & \\
\hline Age (years) & $55.6 \pm 12.3$ & $53.3 \pm 12.6$ & 0.862 \\
\hline Duration after operation (days) & $15.7 \pm 10.2$ & $13.8 \pm 11.3$ & 0.643 \\
\hline Corticosteroid use & 3 & 5 & 0.674 \\
\hline Radiation treatment & 5 & 5 & 0.696 \\
\hline Motricity index & $72.6 \pm 7.8$ & $72.8 \pm 8.6$ & 0.792 \\
\hline \multicolumn{4}{|l|}{ Diagnosis } \\
\hline Meningioma & 1 & 5 & \\
\hline Low grade glioma & 1 & 1 & \\
\hline Ependymoma & 1 & 0 & \\
\hline Glioblastoma & 3 & 2 & \\
\hline CNS lymphoma & 1 & 0 & \\
\hline Metastatic tumor & 2 & 2 & \\
\hline Craniopharyngioma & 0 & 1 & \\
\hline Choroid plexus papilloma & 1 & 0 & \\
\hline Neuroma & 0 & 1 & \\
\hline Site of lesion (right : left : both) & $5: 2: 3$ & $7: 3: 2$ & \\
\hline \multicolumn{4}{|l|}{ Hypnotics } \\
\hline Zolpidem 10 mg & 7 & & \\
\hline Trazodone 25 mg & 3 & & \\
\hline
\end{tabular}

Values are presented as mean \pm standard deviation or number unless otherwise indicated. $\mathrm{M}$ : male, F : female, CNS : central nervous system

Table 2. Comparison of sleep status

\begin{tabular}{|c|c|c|c|c|c|}
\hline & \multicolumn{4}{|c|}{ Insomnia group } & \multirow{3}{*}{$\begin{array}{c}\text { Control group } \\
\text { Score }\end{array}$} \\
\hline & \multicolumn{2}{|c|}{ Before medication } & \multicolumn{2}{|c|}{ After medication } & \\
\hline & Score & $p$-value* & Score & $p$-value ${ }^{\dagger}$ & \\
\hline$|S|$ & $12.1 \pm 4.1$ & $<0.001^{\ddagger}$ & $8.8 \pm 2.8$ & $0.022^{\ddagger}$ & $3.1 \pm 2.2$ \\
\hline PSQI & $10.4 \pm 2.8$ & $<0.001^{\ddagger}$ & $7.1 \pm 2.8$ & $0.010^{\ddagger}$ & $3.3 \pm 1.7$ \\
\hline SSS & $4.0 \pm 1.5$ & $0.002^{\ddagger}$ & $3.3 \pm 0.8$ & 0.227 & $2.1 \pm 1.4$ \\
\hline ESS & $7.4 \pm 4.6$ & 0.070 & $4.4 \pm 2.8$ & $0.004^{\ddagger}$ & $3.7 \pm 2.3$ \\
\hline
\end{tabular}

Values are presented as mean \pm standard deviation. *Comparison of data from the Insomnia group and baseline data from the Control group, using the Mann-Whitney $\mathrm{U}$ test. ${ }^{\dagger}$ Comparison of data before and after hypnotic medication treatment in the Insomnia group, using the Wilcoxon singed rank test. ${ }^{\ddagger} p<0.05$. ISI : Insomnia Severity Index, PSQI : Pittsburg Sleep Quality Index, SSS : Stanford Sleepiness Scale, ESS : Epworth Sleepiness Scale 
test, $p<0.05$ ) (Table 3 ). In contrast, in the control group, only the scores on the first VRT trial were improved after 3 weeks (Wilcoxon signed rank test, $p<0.05$ ), while other scores were not changed (Wilcoxon signed rank test, $p>0.05$ ) (Table 3 ).

\section{Depression}

At baseline, BDI scores in the Insomnia group were significantly higher than those in the control group (Mann-Whitney $\mathrm{U}$ test, $p<0.001)$. When the severity of depression was compared between baseline and follow-up, patients in the insomnia group showed significant improvements after the followup (Wilcoxon signed rank test, $p<0.05$ ), but the control group patients did not $(p>0.05)$ (Table 3$)$.

\section{DISCUSSION}

In this study, we examined the effects of hypnotics on sleep quality and cognition in patients who had undergone surgery for brain tumor resection. Additionally, we evaluated changes in the patients' depressive mood after taking hypnotics.

Sleep disturbances, especially insomnia, are frequently observed in healthy individuals. The prevalence of insomnia has been reported to range between 20\% and 35\% in the general population and between $10 \%$ and $52 \%$ in the elderly ${ }^{11,18)}$. A high prevalence of insomnia has also been observed in patients with neurological diseases. In previous studies, $68 \%$ of patients with stroke and approximately $50 \%$ of brain tumor survivors presented with insomnia ${ }^{22,24)}$. This reported prevalence $(50 \%)$ of insomnia in patients with brain tumors is similar to our study, in which 10 of 25 screened patients (40\%) were diagnosed with insomnia. After administration of hypnotics to the patients with insomnia, at the 3-week follow-up, ISI, PSQI, and ESS scores of the patients with insomnia were significantly improved. ISI and PSQI scores are related to sleep disturbances and quality of sleep, and the ESS score is associ-

Table 3. Comparison of depressive mood and cognitive function

\begin{tabular}{|c|c|c|c|c|c|c|}
\hline & \multicolumn{3}{|c|}{ Insomnia group } & \multicolumn{3}{|c|}{ Control group } \\
\hline & Baseline & Follow-up & $p$-value* & Baseline & Follow-up & $p$-value ${ }^{\dagger}$ \\
\hline MMSE (30) & $24.7 \pm 1.8$ & $26.9 \pm 1.7$ & $0.011^{\ddagger}$ & $25.9 \pm 2.1$ & $27.3 \pm 1.8$ & $0.006^{\ddagger}$ \\
\hline \multicolumn{7}{|l|}{ Attention } \\
\hline VCPT (135) & $103.6 \pm 28.7$ & $126.4 \pm 24.6$ & $0.014^{\ddagger}$ & $132.8 \pm 5.6$ & $133.4 \pm 2.9$ & 0.902 \\
\hline ACPT (135) & $113.0 \pm 21.2$ & $127.6 \pm 17.6$ & $0.012^{\ddagger}$ & $128.2 \pm 9.5$ & $130.6 \pm 4.6$ & 0.345 \\
\hline DSFT (8) & $4.1 \pm 1.3$ & $4.9 \pm 1.5$ & 0.202 & $5.2 \pm 1.2$ & $4.6 \pm 1.4$ & 0.144 \\
\hline DSBT (8) & $2.6 \pm 0.6$ & $3.2 \pm 1.3$ & 0.128 & $3.5 \pm 1.2$ & $3.1 \pm 0.7$ & 0.524 \\
\hline VSFT (8) & $3.6 \pm 1.2$ & $5.2 \pm 1.2$ & $0.047^{\ddagger}$ & $4.1 \pm 1.7$ & $3.9 \pm 1.1$ & 0.462 \\
\hline VSBT (8) & $3.4 \pm 1.7$ & $3.1 \pm 1.1$ & 0.344 & $3.1 \pm 1.4$ & $3.2 \pm 0.8$ & 0.465 \\
\hline \multicolumn{7}{|l|}{ Memory } \\
\hline \multicolumn{7}{|l|}{ AVLT (15) } \\
\hline 1st trial & $4.0 \pm 2.1$ & $4.6 \pm 2.3$ & 0.161 & $3.2 \pm 2.1$ & $5.0 \pm 2.8$ & 0.197 \\
\hline 5th trial & $7.1 \pm 2.2$ & $6.9 \pm 4.0$ & 0.902 & $8.1 \pm 4.1$ & $9.2 \pm 3.7$ & 0.104 \\
\hline Delay trial & $2.9 \pm 3.6$ & $4.5 \pm 3.3$ & 0.493 & $4.4 \pm 3.2$ & $4.4 \pm 5.2$ & 0.285 \\
\hline \multicolumn{7}{|l|}{ VRT (15) } \\
\hline 1st trial & $6.7 \pm 2.3$ & $8.8 \pm 2.3$ & $0.033^{\ddagger}$ & $6.3 \pm 3.7$ & $9.2 \pm 1.5$ & $0.021^{\ddagger}$ \\
\hline 5th trial & $7.6 \pm 2.5$ & $8.9 \pm 2.8$ & $0.047^{\ddagger}$ & $9.5 \pm 3.7$ & $9.6 \pm 1.5$ & 0.705 \\
\hline Delay trial & $7.3 \pm 4.1$ & $8.1 \pm 4.6$ & 0.528 & $6.8 \pm 5.9$ & $9.8 \pm 2.8$ & 0.080 \\
\hline BDI (63) & $24.4 \pm 13.3$ & $18.9 \pm 11.1$ & $0.008^{\ddagger}$ & $6.4 \pm 8.5$ & $6.9 \pm 8.9$ & 0.552 \\
\hline
\end{tabular}

Values are presented as mean \pm standard deviation. ${ }^{*}$ Comparison between baseline and follow-up in the Insomnia group. ${ }^{\dagger}$ Comparison between baseline and follow-up in the Control group. ${ }^{\ddagger} p<0.05$, Wilcoxon signed rank test. MMSE : mini-mental status examination, VCPT : visual continuous performance test, ACPT : auditory continuous performance test, DSFT : digit span forward test, DSBT : digit span backward test, VSFT : visual span forward test, VSBT : visual span backward test, AVLT : auditory verbal learning test, VRT : visual recognition test, BDI : beck depression inventory 
ated with daytime sleepiness. Our results therefore indicate that hypnotics can improve quality of sleep and also reduce sleep disturbance and daytime sleepiness in patients with insomnia after brain tumor resection.

At baseline, sleep-deprived patients' VCPT and ACPT mean scores were lower than those of controls. At the follow-up in our study, the scores for the VCPT, ACPT, VSFT, and VRT (1st and 5th trials) were improved. The VCPT, ACPT, and VSFT are tests that measure attention abilities, and the VRT reflects memory function ${ }^{15)}$. No decline was detected in any of the tests for evaluating cognitive functions. Considering these results, the lower VCPT and ACPT baseline scores for patients with insomnia indicate that these patients had a lower capacity for attention than patients without insomnia. In addition, the increase in some of the cognitive function test scores (with no simultaneous declines in other scores) indicates that the use of hypnotics for managing insomnia does not disturb cognitive function in patients with brain tumors, and our results demonstrate in fact the possibility of a positive effect of hypnotics on cognitive function in patients with brain tumors, especially attention and memory functions. The different baseline results in the two groups and the positive effect on cognitive function in the insomnia group are consistent with the findings of previous studies indicating that sleep deprivation causes impairments in attention and memory functions and that the management of insomnia improves those functions ${ }^{1,9,25)}$.

We also observed that patients with insomnia had higher BDI scores than controls, with a mean value at baseline of 6.4 in the control group and 24.4 for the patients with insomnia. A higher BDI score is indicative of more severe depression, suggesting that the patients with insomnia experienced more severe depressive moods. Several studies have reported a close correlation between insomnia and depression ${ }^{3,32}$. Likewise, in our study, the depressive symptoms in the insomnia group seemed to stem from the insomnia itself, as treatment with hypnotics reduced the symptoms of insomnia, which in turn relieved the depressive mood. Regarding the benefits of pharmacological treatment of depression, Rooney and Grant ${ }^{29)}$ conducted a meta-analysis, and reported that the effects of medication cannot be determined yet, because there are no high-quality studies that have examined the value of pharmacological treatment of depression in patients with brain tumors. Additional well-designed studies are needed to address this issue.

\section{CONCLUSION}

In this study, the quality of sleep of patients after brain tumor resection who experienced insomnia was initially poor but significantly improved after administering hypnotic medication. In addition, our results suggest that hypnotics attribute, at least in part, to the improvement of cognitive impairments and depressive moods in patients after surgery for brain tumor resection. The appropriate use of hypnotics is therefore recommended for patients experiencing insomnia after brain tumor operations. To the best of our knowledge, this is the first study that evaluates the effectiveness of hypnotics in patients with brain tumors with insomnia. However, several limitations of this study should be considered. First, we did not control for sub-type and location of the brain tumor. Second, we did not investigate any potential long-term effects of hypnotics. Third, the number of patients recruited was small. Fourth, our study was conducted without a placebo control group (i.e., a group of patients with insomnia receiving placebo medication). Lastly, we did not consider preoperative comorbid conditions. Therefore, further studies addressing these limitations are necessary to confirm the findings of the current study.

\section{CONFLICTS OF INTEREST}

No potential conflict of interest relevant to this article was reported.

\section{INFORMED CONSENT}

Informed consent was obtained from all individual participants included in this study.

\section{AUTHOR CONTRIBUTIONS}

\author{
Conceptualization : $\mathrm{MHC}$ \\ Data curation : $\mathrm{MHC}$ \\ Formal analysis : MCC, MHC \\ Funding acquisition : $\mathrm{MHC}$ \\ Methodology : MHC
}


Project administration : $\mathrm{MHC}$

Visualization : $\mathrm{MHC}$

Writing - original draft : MCC

Writing - review \& editing : MCC, $\mathrm{MHC}$

\section{ORCID}

Min Cheol Chang https://orcid.org/0000-0002-7629-7213

Min Ho Chun https://orcid.org/ 0000-0001-8666-7225

\section{References}

1. Alhola P, Polo-Kantola P : Sleep deprivation: impact on cognitive performance. Neuropsychiatr Dis Treat 3 : 553-567, 2007

2. Bastien $\mathrm{CH}$, Vallières $\mathrm{A}$, Morin $\mathrm{CM}$ : Validation of the Insomnia Severity Index as an outcome measure for insomnia research. Sleep Med 2 : 297-307, 2001

3. Bei B, Asarnow LD, Krystal A, Edinger JD, Buysse DJ, Manber R : Treating insomnia in depression: insomnia related factors predict long-term depression trajectories. J Consult Clin Psychol 86 : 282-293, 2018

4. Berch DB, Krikorian R, Huha EM : The Corsi block-tapping task: methodological and theoretical considerations. Brain Cogn 38 : 317-338, 1998

5. Buysse DJ, Reynolds CF 3rd, Monk TH, Berman SR, Kupfer DJ : The Pittsburgh Sleep Quality Index: a new instrument for psychiatric practice and research. Psychiatry Res 28 : 193-213, 1989

6. Callens CJ, Meltzer ML : Effect of intelligence, anxiety, and diagnosis on arithmetic and digit span performance on the WAIS. J Consult Clin Psychol 33 : 630, 1969

7. Campbell SS, Murphy PJ. The nature of spontaneous sleep across adulthood. J Sleep Res 16 : 24-32, 2007

8. Chervin RD, Aldrich MS, Pickett R, Guilleminault C : Comparison of the results of the Epworth sleepiness scale and the multiple sleep latency test. J Psychosom Res 42 : 145-155, 1997

9. Chua EC, Fang E, Gooley JJ : Effects of total sleep deprivation on divided attention performance. PLoS One 12 : e0187098, 2017

10. Conners CK, Epstein JN, Angold A, Klaric J : Continuous performance test performance in a normative epidemiological sample. J Abnorm Child Psychol 31 : 555-562, 2003

11. Ford DE, Kamerow DB : Epidemiologic study of sleep disturbances and psychiatric disorders. An opportunity for prevention? JAMA 262 : 14791484, 1989

12. Gapstur R, Gross CR, Ness K : Factors associated with sleep-wake disturbances in child and adult survivors of pediatric brain tumors: a review. Oncol Nurs Forum 36 : 723-731, 2009

13. Gu SY, Chang MC : The effects of $10-\mathrm{Hz}$ repetitive transcranial magnetic stimulation on depression in chronic stroke patients. Brain Stimul 10 : 270-274, 2017
14. Ha KS, Kwon JS, Lyoo IK, Kong SW, Lee DW, Youn T : Development and standardization process, and factor analysis of the computerized cognitive function test system for Korea adults. J Korean Neuropsychiatr Assoc 41 : 551-562, 2002

15. Hoddes E, Zarcone V, Smythe H, Phillips R, Dement WC : Quantification of sleepiness: a new approach. Psychophysiology $10: 431-436,1973$

16. Holbrook AM, Crowther R, Lotter A, Cheng C, King D : Meta-analysis of benzodiazepine use in the treatment of insomnia. CMAJ $162: 225$ 233, 2000

17. Holm KJ, Goa KL : Zolpidem: an update of its pharmacology, therapeutic efficacy and tolerability in the treatment of insomnia. Drugs 59 : 865889,2000

18. Husby $\mathrm{R}$, Lingjaerde 0 : Prevalence of reported sleeplessness in northern Norway in relation to sex, age and season. Acta Psychiatr Scand 81 : 542-547, 1990

19. Jang $\mathrm{SH}$, Chang MC : Motor outcomes of patients with a complete middle cerebral artery territory infarct. Neural Regen Res 8 : 1892-1897, 2013

20. Mefferd RB Jr, Wieland BA, James WE : Repetitive psychometric measures: digit span. Psychol Rep 18 : 3-10, 1966

21. Milner $B$ : Interhemispheric differences in the localization of psychological processes in man. Br Med Bull 27 : 272-277, 1971

22. Müller HL, Handwerker G, Wollny B, Faldum A, Sörensen N : Melatonin secretion and increased daytime sleepiness in childhood craniopharyngioma patients. J Clin Endocrinol Metab 87 : 3993-3996, 2002

23. Ohayon MM, Hong SC : Prevalence of insomnia and associated factors in South Korea. J Psychosom Res 53 : 593-600, 2002

24. Palomäki $H$, Berg $A$, Meririnne $E$, Kaste $M$, Lönnqvist $R$, Lehtihalmes $M$, et al. : Complaints of poststroke insomnia and its treatment with mianserin. Cerebrovasc Dis 15 : 56-62, 2003

25. Pasula EY, Brown GG, McKenna BS, Mellor A, Turner T, Anderson C, et al. : Effects of sleep deprivation on component processes of working memory in younger and older adults. Sleep 41 : zsx213, 2018

26. Provini F, Lombardi C, Lugaresi $E$ : Insomnia in neurological diseases. Semin Neurol $25: 81-89,2005$

27. Query WT, Megran J : Age-related norms for AVLT in a male patient population. J Clin Psychol 39 : 136-138, 1983

28. Roger M, Attali P, Coquelin JP : Multicenter, double-blind, controlled comparison of zolpidem and triazolam in elderly patients with insomnia. Clin Ther 15 : 127-136, 1993

29. Rooney A, Grant R : Pharmacological treatment of depression in patients with a primary brain tumour. Cochrane Database Syst Rev 31 : CD006932, 2013

30. Scharf MB, Mayleben DW, Kaffeman M, Krall R, Ochs R : Dose response effects of zolpidem in normal geriatric subjects. J Clin Psychiatry 52 : 77-83, 1991

31. Schwartz MD, Kilduff TS : The neurobiology of sleep and wakefulness. Psychiatr Clin North Am 38 : 615-644, 2015

32. Taylor DJ : Insomnia and depression. Sleep 31 : 447-448, 2008

33. Zhou ES, Manley PE, Marcus KJ, Recklitis CJ : Medical and psychosocial correlates of insomnia symptoms in adult survivors of pediatric brain tumors. J Pediatr Psychol 41 : 623-630, 2016 